\title{
Reinforcement of ambiguous-cue problem performance under various across trial fixed-ratio schedules*
}

\section{RALPH W. RICHARDS \\ Colorado State Liniversity. Ft. Collins. Colo. 80521}

Two pigeons were trained on the two-choice ambiguous-cue problem that contained two types of trials. On PA trials, choice of $P$ (the positive stimulus) is reinforced and choice of $A$ (the ambiguous stimulus) is not reinforced; on NA trials, choice of $A$ is reinforced and choice of $N$ (the negative stimulus) is not reinforced. Under the various across-trial fixed-ratio (FR) schedules of reinforcement, NA performance remained superior to PA performance. PA performance was poorer under FR 12 than FR 1; NA performance, however, was not affected by the size of the FR. Similarly, only PA performance showed any change as a function of the trial's location within the ratio-it was poorest early in the ratio.

The most widely studied simultaneous discrimination task presents a $\mathrm{S}$ with only two stimuli. Choice of one stimulus, the positive stimulus $(\mathrm{P})$, is reinforced and choice of the other stimulus, the negative stimulus $(N)$, is not reinforced. An interesting variant of this two-choice problem, the ambiguous-cue problem, involves a third stimulus, an ambiguous stimulus (A). Specifically, the ambiguous-cue problem contains two types of trials-PA trials and NA trials. On PA trials, $\mathrm{P}$ and $A$ are simultaneously presented; the choice of $P$ is reinforced and the choice of $A$ is not reinforced. On NA trials, $\mathbf{N}$ and $\mathbf{A}$ are simultaneously presented; the choice of $\mathrm{A}$ is reinforced and the choice of $\mathrm{N}$ is not reinforced. The consistent finding, when the stimuli possess distinctive features, has been that NA trials are learned more quickly and are learned to a higher terminal level than PA trials. This finding has been reported in monkeys (Leary, 1958; Boyer, Polidora, Fletcher, \& Woodruff, 1966; Fletcher, Grogg, \& Garske, 1968; Fletcher \& Garske, 1972; Boyer \& Polidora, 1972), retarded children (Zeaman \& House, 1962; Fletcher et al, 1968), normal children (Fletcher et al, 1968), and most recently in pigeons (Richards, 1973).

In all previous ambiguous-cue studies, the across-trial schedule of reinforcement has been continuous reinforcement. That is, reinforcement was received on every trial if the correct response was made. The present study examined ambiguous-cue problem performance as a function of various fixed ratio (FR) schedules; an FR schedule reinforces every $\mathrm{n}^{\text {th }}$ response. Within the context of the ambiguous-cue problem, an FR across-trial schedule of reinforcement makes reinforcement available on every $\mathrm{n}^{\text {th }}$ trial. The present experiment attempted to determine if ambiguous-cue

*This research was supported in part by BSSG Grant 5 SO 5 RR 07127-03. The author would like to thank David Schwartz and William $M$. Hittesdorf for their assistance in collecting the data. The paper is sponsored by Henry $A$. Cross, who takes full editorial responsibility for it. problem performance could be maintained by such an intermittent schedule and, if so, at what level. Also of interest were possible changes in accuracy and response latency as a function of the trial's location within the ratio, as well as possible differential schedule effects on PA and NA performance.

\section{METHOD \\ Subjects and Apparatus}

The two adult White Carneaux pigeons, maintained at approximately $70 \%$ of their free-feeding weights, had previously received 25 sessions of ambiguous-cue problem training under a schedule of continuous reinforcement. During this prior training (described fully in Richards, 1973) and throughout the present study, the P, N, and A stimulus lights were, respectively: green, red, and orange for S-2333 and red, orange and green for S-2750. These lights illuminated the right and left keys of the standard operant conditioning chamber. The reinforcer also remained $2.5 \mathrm{sec}$ access to mixed grain.

\section{Procedure}

Both keys were illuminated at the beginning of a trial so as to present one of the following types of trials (a) PA with $P$ on the right key, (b) PA with P on the left key, (c) NA with $\mathrm{N}$ on the right key, and (d) NA with $\mathrm{N}$ on the left key. Order of trials was random, with two restrictions. First, each of the four types of trials was presented five times in each block of 20 trials. Second, none of the types of trials could be presented more than three times in succession.

Table 1 summarizes the reinforcement contingencies during the present experiment. Each trial in all phases terminated after one response. An incorrect response produced a 10-sec timeout, during which the keys were darkened and responding was nonfunctional. A correct response during a nonreinforcement trial also produced a timeout; however, during the FR 3 (with FB) and FR 12 (with FB) conditions, it also produced a 1/8-sec operation and illumination of the food magazine. This duration was too brief to allow consumption of any grain and served as feedback for correct, but nonreinforced, responses. Both correct and incorrect responses advanced the ratio, which reset on receipt of the reinforcer. Reinforcement was always followed by a 7.5-sec timeout. Identical timeout and intertrial interval

Table 1

Summary of Procedure

\begin{tabular}{llcc}
\hline & Schedule & $\begin{array}{c}\text { Number of } \\
\text { Trials Per } \\
\text { Session* }\end{array}$ & $\begin{array}{c}\text { Number } \\
\text { of } \\
\text { Sessions }\end{array}$ \\
\hline & FR 3 & 60 & 25 \\
S-2333 & FR 3 (with FB) & 120 & 20 \\
& FR 3 & 120 & 25 \\
& FR 6 & 120 & 25 \\
& FR 12 & 240 & 50 \\
& FR 1 & 40 & 30 \\
S-2750 & FR 3 & 60 & 25 \\
& FR 6 & 120 & 20 \\
& FR 12 12 (with FB) & 120 & 20 \\
& FR 12 & 120 & 25 \\
& FR 12 & 120 & 1 \\
& FR 1 & 240 & 49 \\
\hline
\end{tabular}

* Several sessions were terminated before their scheduled completion because of. long pauses. in responding. 


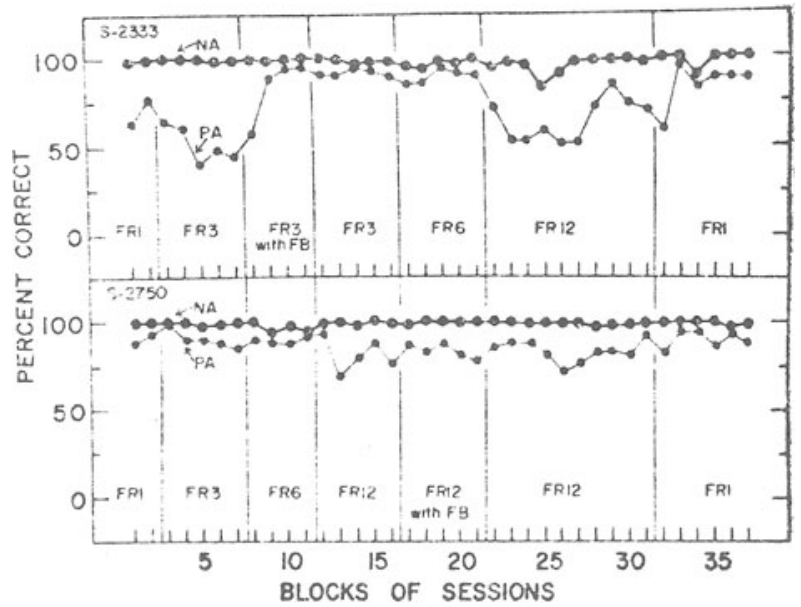

Fig. 1. Mean performance on PA and NA trials as a function of the various across-trials reinforcement schedules.

durations were employed during the Ss' previous training. Also, as in prior training, the houselight remained illuminated throughout the session, except as noted below.

During Sessions 126-135 of the present study, the center key was illuminated by a white light. A response on this key produced a 30 -sec blackout, but did not advance the ratio. Since this key was responded to infrequently (except during Session 126 by S-2750), no further mention of it will be made.

\section{RESULTS}

The last half of each session's data is included in Fig. 1, which shows the Ss' performance during all phases of the present experiment, as well as during the final 10 sessions of previous training under a FR 1 schedule. In general, five sessions are included in each block; the data from the occasional sessions that were terminated early are omitted. Both Ss showed perfect, or near-perfect, performance on NA trials across all phases, and, while their performance on PA trials also frequently attained high levels, PA performance remained inferior to NA performance across the 175 sessions of the present study. Figure 1 also shows that on first exposure to the FR 3 schedule, the PA performance of S-2333 gradually dropped to a level slightly below chance; the addition of feedback (FB) following each correct response, on nonreinforced trials, was accompanied by a gradual improvement, until an accuracy level of about $95 \%$ was attained. With the subsequent removal of $\mathrm{FB}$, only slightly poorer levels of PA performance were evidenced on the FR 3 and on the subsequently introduced FR 6 schedule. When the scheldue was further increased to an FR 12, PA performance again fell to a near chance level; however, extended training produced substantial improvement, with an accuracy level of about $75 \%$ finally being attained. Subsequent exposure to FR 1 produced a terminal PA performance level of about $85 \%$, which, although greater than that during the FR 12 phase, was slightly below that during the FR 6 phase and the second and third phases of FR 3. The PA performance of S-2750 was much less affected by increases in the FR requirement. Accuracy was maintained at between $85 \%$ and $95 \%$ during FR 1, FR 3, and FR 6, and at only a slightly lower level during FR 12. The addition of FB during FR 12 produced no change in S-2750's performance.

Figure 2 presents PA and NA performance as a function of trial location within the FR 12 schedule. This figure is based on the final 10 sessions of FR 12 reinforcement, and, again, represents only the last half of the completed sessions. Performance on NA trials was at a near perfect level at each location within the ratio. PA performance, however, was lowest during the early portion of the ratio for both S-2333 (60\%) and S-2750 (76\%). The PA performance of S-2333 stabilized at about $77 \%$ during both the middle and late portions of the ratio. S-2750, however, showed increased accuracy through the middle (88\%) and late $(92 \%)$ portions of the ratio.

In marked contrast to the results reported in Figs. 1 and 2 , the analysis of response latencies during the final 10 sessions of FR 12 reinforcement failed to show any differential effect due to the type of trial. Thus, the within-ratio latencies that are presented in Fig. 3 represent a pooling of PA and NA trials. Although the effect is much larger for S-2750, both Ss show the longest mean latency on Trial 1, with subsequent latencies decreasing (to an asymptote of about $.02 \mathrm{~min}$ ) as the proximity to reinforcement increased.

The mean response latencies on the first trial within each ratio during the last half of each of the final 10

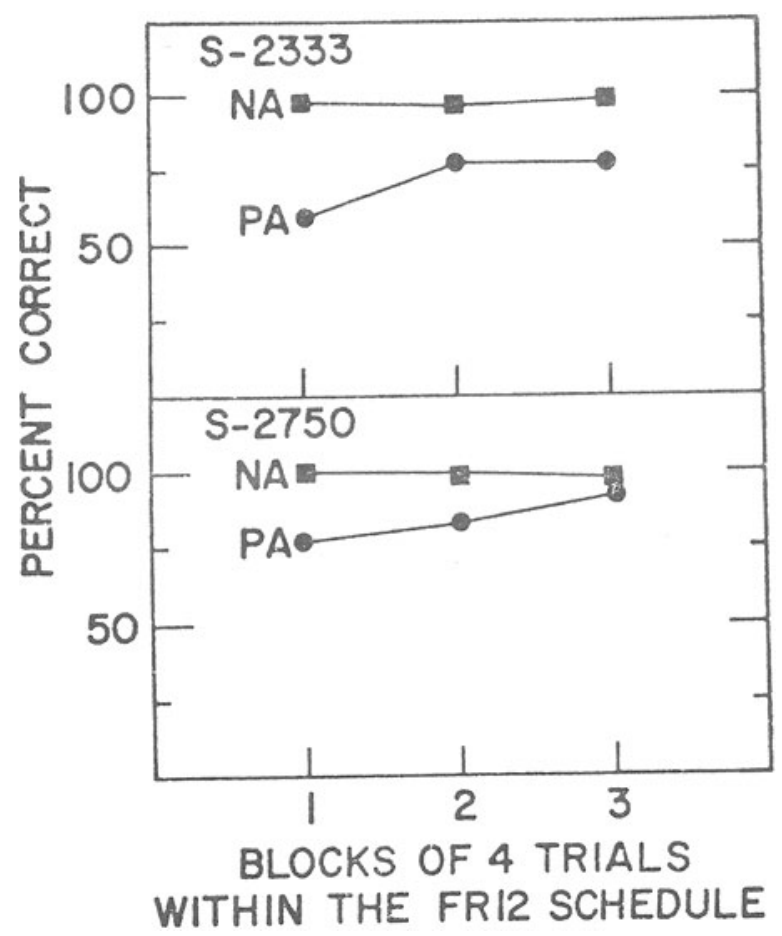

Fig. 2. Mean performance on PA and NA trials over the last half of each of the final 10 sessions of FR 12 as a function of trial location within the ratio. 
sessions under FR 1, FR 3, FR 6, and FR 12 were, respectively: $.01, .02, .10$, and $.43 \mathrm{~min}$ for S-2750, and $.02, .05, .06$, and $.04 \mathrm{~min}$ for S-2333. Thus, only S-2750 showed increasing latencies on the first trial of the ratio as the ratio size increased.

\section{DISCUSSION}

The continued superiority of NA performance over PA performance throughout the present study is in agreement with apparently every other published ambiguous-cue experiment that has employed distinctive stimuli (Leary, 1958; Zeaman \& House, 1962; Boyer et al, 1966; Fletcher et al, 1968; Fletcher \& Garske, 1972; Boyer \& Polidora, 1972; Richards, 1973). Differential sensitivity of PA and NA performances to the experimental manipulations of the present study was also noted in the present experiment. The performance on NA trials was not affected by increasing the FR size, providing or removing feedback for correct, but nonreinforced, responses, nor was it a function of the trial's location within the FR. In marked contrast, PA performance was affected by the FR size-both Ss showed poorer performance on the FR 12 than on the FR 1. Feedback seemed to improve the PA performance of one of the Ss. And PA performance was affected by the trial's location within the

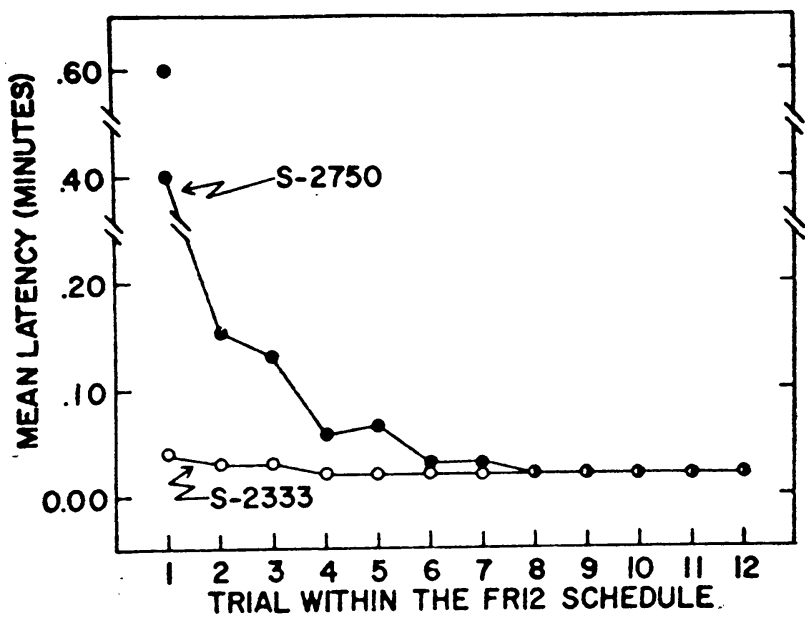

Fig. 3. Mean response latencies over the last half of each of the final 10 sessions of FR 12 as a function of trial location within the ratio. The upper data point on the first trial for S-2750 is based on all latencies during the last half of each of the final 10 sessions; the lower data point excludes one of the latencies that had a duration of more than $13 \mathrm{~min}$. ratio; performance on the PA trials, in agreement with that on various other discrimination tasks [such as, a simple three-choice discrimination (Zeiler, 1968) and a matching-to-sample task (Nevin, Cumming, \& Berryman, 1963; Mintz, Mourer, \& Weinberg, 1966)] was poorest early in the ratio.

Response latencies within the FR 12, however, did not differ in terms of the type of trial, although they did differ in terms of the trial's proximity to reinforcement. As with simple operants (e.g., Ferster \& Skinner, 1957) and various other types of discrimination problems (e.g., Zeiler, 1968; Nevin, et al, 1963; Mintz et al, 1966), the first response of the ratio had the longest latency. In contrast with these previous FR studies, which, in general, have found a constant response rate or latency once the first response is executed, subsequent response latencies in the present study gradually decreased (this was most clearly seen in $S-2750$ ). Finally, one $S$ did show the increasing functional relationship between the latency of the first response within the ratio and FR size, which has typically been reported with simple operants (e.g., Felton \& Lyon, 1966).

\section{REFERENCES}

Boyer, W. N., Polidora, V. J., Fletcher, H. J., \& Woodruff, W. Monkeys' performance on ambiguous-cue problems. Perceptual \& Motor Skills, 1966, 22, 883-888.

Boyer, W. N., \& Polidora, V. J. An analysis of the solution of PAN ambiguous-cue problems by rhesus monkeys. Learning \& Motivation, 1972, 3, 325-333.

Felton, M., \& Lyons, D. O. The post-reinforcement pause. Journal of the Experimental Analysis of Behavior, 1966, 9, 131-134.

Ferster, C. B., \& Skinner, B. F. Schedules of reinforcement. New York: A ppleton-Century-Croft, 1957.

Fletcher, H. J., \& Garske, J. P. Response competition in monkeys' solution of PAN ambiguous-cue problems. Learning \& Motivation, 1972, 3, 334-340.

Fletcher, H. J., Grogg, T. M. \& Garske, J. P. Ambiguous-cue problem performance of children, retardates, and monkeys. Journal of Comparative \& Physiological Psychology, 1968, 66, 477-482.

Leary, R. W. The learning of ambiguous-cue problems by monkeys. American Journal of Psychology, 1958, 71, 718-724.

Mintz, D. E., Mourer, D. J., \& Weinberg, L. S. Stimulus control in fixed ratio matching-to-sample. Journal of the Experimental Analysis of Behavior, 1966, 9, 627-630.

Nevin, J. A., Cumming, W. W., \& Berryman, R. Ratio reinforcement of matching behavior. Journal of the reinerimental Analysis of Behavior, 1963,6, 149-154.

Richards, R. W. Performance of the pigeon on the ambiguous-cue problem. Bulletin of the Psychonomic Society, $1973,6,445-447$.

Zeaman, D. \& House, B. J. Approach and avoidance in discrimination learning of retardates. Child Development, 1962, 33, 355-372.

Zeiler, M. Stimulus control with fixed ratio reinforcement. Journal of the Experimental Analysis of Behavior, 1968, 11, 107-115.

(Received for publication August 30, 1973.) 